\title{
Experimental setup for performance characterization of a jet pump with varying angles of placement and depth
}

\author{
Rit Nanda $\cdot$ Shashank Gupta $\cdot$ Ajit Kumar N Shukla
}

Received: 5 August 2011/Accepted: 18 September 2011/Published online: 5 October 2011

(C) The Author(s) 2011. This article is published with open access at Springerlink.com

\begin{abstract}
In an oil field, when the crude cannot come to the surface on its own due to either low pressure differential or the fluid properties like high viscosity, artificial lift technology has to be used. The jet pump is one of the artificial lift techniques considered to be better than other lift systems because of no moving parts and simple working principle. This makes it easy to monitor and regulate. It works on the fluid power hence it can be placed at greater depths and at varying angles with respect to the horizontal. In a field, some wells are deeper than the others, while some are deviated. The performance of a jet pump is different for different cases as the parameters affecting it are the depth and the angle of placement. These aspects with respect to the working of the jet pump need to be investigated and demonstrated. Hence, an effort has been made in this paper to design an experimental setup by which the pump efficiency with respect to depths and angles of placement can be easily determined. The experimental setup uses a jet pump driven by an electric motor of $2.5 \mathrm{~kW}$ along with a measurement setup. The jet pump was mounted on a hinged fixture and injection fluid from a centrifugal pump was used. The gate valve was used to throttle the flow for injection and create resistance across the delivery line of the jet pump as a measure of increasing depth. These flow rates were used to characterize the performance of the jet pump.
\end{abstract}

Keywords Artificial lift · Jet pump · Discharge flow rate . Injection flow rate

R. Nanda · S. Gupta · A. K. N. Shukla $(\bowtie)$

School of Petroleum Technology, Pandit Deendayal Petroleum

University, Gandhinagar, Gujarat, India

e-mail: ajit.shukla@spt.pdpu.ac.in

\author{
List of symbols \\ $Q_{\mathrm{i}} \quad$ Injection flow rate, $\mathrm{m}^{3} / \mathrm{s}$ \\ $Q_{\mathrm{s}} \quad$ Suction flow rate, $\mathrm{m}^{3} / \mathrm{s}$ \\ $Q_{0} \quad$ Discharge flow rate, $\mathrm{m}^{3} / \mathrm{s}$ \\ $P_{\mathrm{d}} \quad$ Discharge pressure, bar \\ $\eta \quad$ Efficiency, \%
}

\section{Introduction}

Artificial lift is a system that lifts the fluid from bottom of the well to the surface artificially. In a self flowing well, the pressure at the bottom is sufficient to naturally lift the fluid to the surface. But once the crude is produced the pressure at the bottom of the wellbore decline makes it unable to come to the surface of its own. High viscosity of the crude is another reason due to which the wells do not self flow (Takacs 2005).

One of the artificial lift techniques is to use jet pump. Here, the fluid is circulated through a chamber, housing a foot valve and a venturi which causes the pressure drop when the fluid is injected before the venturi. It sets the flow in the pipeline housing the foot valve. Armstrong (2010) in his study has documented the working of jet pump in terms of principle and applications. Kwon et al. (2002) has evaluated the chamber shape effect on the efficiency of jet pump.

Irrespective of the chamber shape, it is important to check the performance of a jet pump for wellbore deviation and depth parameters, as the well bore is seldom vertical. To characterize the performance of the jet pump, various tests have been suggested by Lal (2000). The performance characterization of jet pump for various nozzle angles has been studied before (New 2009), but the effect of angle of 
placement for a vertical nozzle has not been reported. This can be checked by the experimental setup and recommendation can be made to study the effect on pump performance by angle and depth.

In the following sections, an experimental setup has been explained to measure the efficiency of a jet pump with varying angles of placement and depth. The effect of diameter and nozzle-throat area ratio and its effects have been studied before by Lima Neto 2011 and the same has not been reported here.

\section{Assembly of a jet pump test rig (JPTR)}

The following procedure was followed while assembling a jet pump test rig. The materials and equipments used have been detailed in relevant steps as follows:

- A $2.5 \mathrm{~kW}$ powered jet pump was installed on a tank of 2001 capacity. The tank was filled to the level of $400 \mathrm{~mm}$ for testing purpose as shown in Fig. 1.

- A collecting tank of approximately 431 capacity was installed for measurement of flow rate from the jet pump. Transparent graded tubing was used to see the level of water in the collecting tank and simultaneous measurement for time duration was done to calculate the discharge flow rate.

- A hinged fixture was developed with slots for $90^{\circ}, 60^{\circ}$, $45^{\circ}, 30^{\circ}$ and $0^{\circ}$ hold angles. The jet pump was mounted on the hinged arm and the fixed arm was kept as base. Initially, the hinged arm was kept perpendicular to the fixed arm, so that the jet pump was positioned vertical to perform the test.

- The pump was primed and electric motor powering the injection line was switched on.

- The gate valve fitted on the injection line was used to control flow rates. The flow rate was measured by the

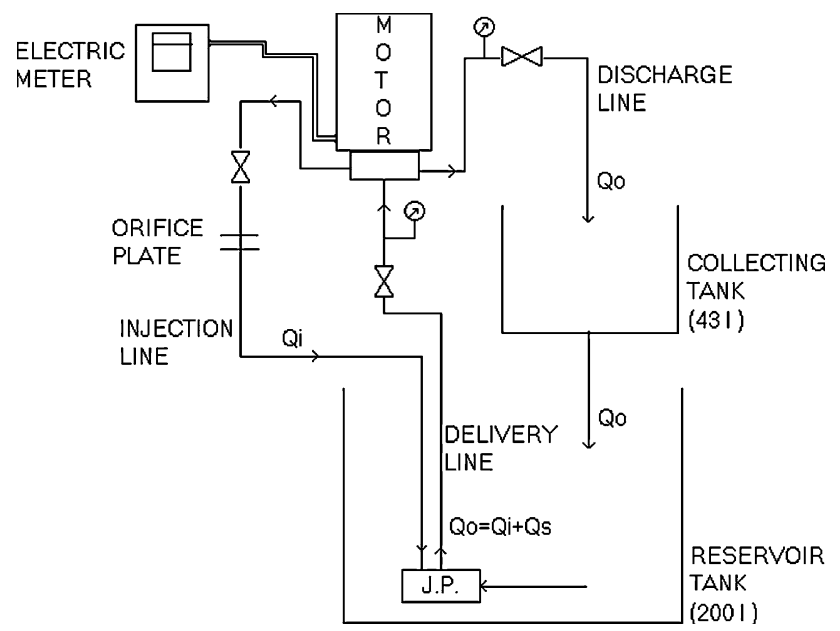

Fig. 1 Schematic of the experimental setup pressure drop across the orifice plate fitted in the injection line as shown in Fig 1. Mercury manometer was used to measure the pressure drop across the orifice.

- The discharge valve in the discharge line was used to control the discharge flow rate and pressure gauge was used to measure the discharge pressure. Similarly, suction pressure gauge was used to measure the pressure in the delivery line of the jet pump.

- The performance characteristic of jet pump was obtained in vertical position at constant injection rates by varying discharge flow rates. Similarly, the performance test was done at two other angles of placement, $45^{\circ}$ and at horizontal position.

\section{Experimentation and computation}

To evaluate the performance characteristics, values of flow rates are calculated for various angles of placement and depths. The computation process was carried out as under:

- The efficiency was calculated at five different points of opening of gate valve at set injection rate. The point of best efficiency was obtained through this which signifies the operating point at given injection rate. Similarly, the test was repeated for other positions.

- Now the angle of placement was changed and the efficiency was calculated for the above injection flow rate and the corresponding point of best efficiency was determined. It was found that the efficiency dropped when the angle of placement was changed from vertical to horizontal position.

- When the gate valve fitted on delivery line was closed, a reduction in discharge pressure signified the effect of increased depth and the values of efficiencies were measured at each angle of placement. It was found that by increasing the head over the pump the efficiency increased.

- The above steps were checked for repeatability and the operating point was found at the nearly same point in each case.

- Furthermore, the range of efficiency in each measurement showed repeatability with previous measurements for the same operating conditions.

\section{Results and discussion}

The performance characteristics of jet pump are reported at two conditions:

a. Various angles of placement

b. Various depths of placement at different angles 
Table 1 shows that at constant injection flow rate and discharge head the efficiency of jet pump is higher when it is placed vertically than when it is placed horizontally. The flow rate at vertical position is around $9 \%$ greater than when it is placed in horizontal position. The reason to this is attributed to greater buoyancy force due to submergence but having dead weight of the valve same. This follows the linear relationship as at $45^{\circ}$, midway the corresponding increase is around 5\% only. It further shows that suction flow rate is greater when it is positioned vertically and the magnitude is around $22 \%$ higher while at $45^{\circ}$, again it follows the linear relationship reducing to $11 \%$ rise from the minimum flow rate. Corresponding to this, there is a $5 \%$ difference in efficiency when the jet pump is placed in vertical position and horizontal position. The analyses here consider single phase fluid with no dissolved gas in it. As the main flow take the fluid upward beyond the venturi, the pressure remains positive and is sufficient for maintaining only the flow. It does not carry any hydrodynamic action as at fully closed position of delivery valve the injection line act as the circulating line without harming the pump.

Table 2 shows that at vertical position of the jet pump the efficiency of the pump decreases if the depth of the pump is decreased. It also shows that the discharge flow rate increases when depth is increased. A $10 \%$ increase in flow caters to $15 \%$ increase in head over the pump. This is explained as with larger head it provides larger length of the pipe which carries kinetic energy of the fluid for longer time. Hence, jet pump works better at higher depth and deliver large amount of fluid. At this condition, the corresponding increase in suction flow rate is of order of

Table 1 Influence of angle of placement on pump performance

\begin{tabular}{llllll}
\hline $\begin{array}{l}\text { Angle of } \\
\text { placement }\end{array}$ & $\begin{array}{l}\text { Total } \\
\text { head } \\
\text { (bar) }\end{array}$ & $\begin{array}{l}Q_{\mathrm{o}} \\
\left(\mathrm{m}^{3} / \mathrm{s} \times 10^{3}\right)\end{array}$ & $\begin{array}{l}Q_{\mathrm{i}} \\
\left(\mathrm{m}^{3} / \mathrm{s} \times 10^{3}\right)\end{array}$ & $\begin{array}{l}Q_{\mathrm{s}} \\
\left(\mathrm{m}^{3} / \mathrm{s} \times 10^{3}\right)\end{array}$ & $\begin{array}{l}\eta \\
(\%)\end{array}$ \\
\hline Vertical & 0.51 & 0.669 & 0.361 & 0.308 & 46 \\
$45^{\circ}$ & 0.51 & 0.641 & 0.361 & 0.280 & 44 \\
Horizontal & 0.51 & 0.612 & 0.361 & 0.252 & 41 \\
\hline
\end{tabular}

Table 2 Influence of depth and positions on pump performance

\begin{tabular}{llllll}
\hline $\begin{array}{l}\text { Angle of } \\
\text { placement }\end{array}$ & $\begin{array}{l}\text { Total } \\
\text { head } \\
(\mathrm{bar})\end{array}$ & $\begin{array}{l}Q_{\mathrm{o}} \\
\left(\mathrm{m}^{3} / \mathrm{s} \times 10^{3}\right)\end{array}$ & $\begin{array}{l}Q_{\mathrm{i}} \\
\left(\mathrm{m}^{3} / \mathrm{s} \times 10^{3}\right)\end{array}$ & $\begin{array}{l}\mathrm{Qs} \\
\left(\mathrm{m}^{3} / \mathrm{s} \times 10^{3}\right)\end{array}$ & $\begin{array}{l}\eta \\
(\%)\end{array}$ \\
\hline Vertical & 1 & 0.583 & 0.361 & 0.222 & 38 \\
& 0.85 & 0.521 & 0.361 & 0.160 & 31 \\
$45^{0}$ & 1 & 0.625 & 0.361 & 0.264 & 42 \\
& 0.85 & 0.563 & 0.361 & 0.202 & 36 \\
Horizontal & 1 & 0.585 & 0.361 & 0.224 & 38 \\
& 0.85 & 0.531 & 0.361 & 0.171 & 32 \\
\hline
\end{tabular}

$30-38 \%$. This means that at greater depth in this case $15 \%$ increase the force due to buoyancy make the valve opening easy accommodating larger flux of fluid to get in. This leads to 6 to 7 basis point increase in efficiency from its base point. Likewise, the efficiency and discharge flow rate curiously have nearly the same magnitude at horizontal position and vertical position. It is because once the flow commences through the foot valve, of the two components: vertical and horizontal flows, one component of flow becomes zero while the other is active. But when the jet pump is at $45^{\circ}$ both the component remains and there is less fluid loss. This ensures that efficiency is marginally better for $45^{\circ}$ at greater depth. In this case it is around $4 \%$ better. All throughout the experiment, the injection rate is kept constant so its influence is not accounted here with. Nevertheless, it is expected that at larger injection power the above outcome will be magnified.

\section{Conclusion}

The experimental setup so assembled was successful in creating a system by which the performance of the jet pump was characterized at different angles of placement and depth. To enable this, unique fixture is designed. The stress of the work is on experimental setup for performance evaluation of a jet pump where in practical field it is difficult to make such an analysis. The success of work lies in creating a system of arrangement through which influence of varying angle of placement and depth is evaluated. The range of variation of flow at vertical position to horizontal position changed the discharge flow rate from 0.308 to $0.252 \mathrm{l} / \mathrm{s}$ which is order of $22 \%$ increase. The efficiency was a maximum of $46 \%$ when jet pump was placed vertically. It dropped to $42 \%$ at $45^{\circ}$ when placed at greater depth. When there was $15 \%$ decrease in discharge pressure, there was around $10 \%$ decrease in the flow rate. The above variations are calculated at constant operating injection flow rates. The facility so generated can be used for future research work on various aspects of jet pump by experimentation. This study presents two interesting outcomes as a result of testing the influence of angle of placement and depth over jet pump: at constant depth jet pump works better at vertical position but when it is under the influence of both depth and angle of placement, incline position is better once flow is commenced. It is also being an experimental experience that jet pump performance is a function of valve seating design and active pressure control is expected to work better than the gravity and buoyancy settled valve. At shallow depth, jet pump is expected to perform better and the maximum limit for immersion will be checked against vapour pressure for the working fluid. 
The experimental aspect of the setup developed is expected to be extensible used for future work and create snow ball effect in terms of the arrangement for varying angle of the placement and depth. The authors propose that further research can be carried out by varying the angle of placement and depth for the following parameters: different nozzle angles, different chambers shapes, varying diameters, area ratio and positive and negative suction heads (Hammoud 2006).

Acknowledgments The authors wish to acknowledge the use of facility and technical help provided by the personnel at the fabrication technology laboratory, Pandit Deendayal Petroleum University, Gandhinagar, India, without whom this experiment could not have been performed.

Open Access This article is distributed under the terms of the Creative Commons Attribution License which permits any use, distribution and reproduction in any medium, provided the original author(s) and source are credited.

\section{References}

Armstrong OP (2010) Summary of oil jet pump principles and applications. SPE, Dallas

Hammoud AH 2006 Effect of design and operational parameters on jet pump performance. In: 4th WSEAS International Conference on fluid mechanics and aerodynamics, p 245-252. http://www.wseas.us/e-library/conferences/2006elounda2/papers/ 538-122.pdf. Accessed 29 Sept 2011

Kwon OB, Kim MK, Kwon HC, Bae DS(2002) Two-dimensional numerical simulations on the performance of an annular jet pump. J Vis 5(1):21-28. doi:10.1007/BF03182599

Lal J (2000) Hydraulic machines (including fluidics), testing characteristics of hydraulic machines. Metropolitan Book Company Pvt Ltd, New Delhi, pp 444-445

Lima Neto IE (2011) Maximum suction lift of water jet pumps. J Mech Sci Tech 25(2):391-394

New TH (2009) An experimental study on jets issuing from elliptic inclined nozzles. Exp Fluids 46(6):1139-1157. doi: 10.1007/s00348-009-0622-9

Takacs G (2005) Gas lift manual, inflow performance of oil wells. PennWell Corp, Tulsa, p 21 